\title{
Sleep Posture Affects Sleep Parameters Differently in Young and Senior Japanese as Assessed by Actigraphy
}

\author{
Akiko Nojiri' ${ }^{1}$, Chikako Okumura ${ }^{1}$, Yushi Ito ${ }^{2}$ \\ ${ }^{1}$ Faculty of Rehabilitation, Kumamoto Health Science University, Kumamoto, Japan \\ ${ }^{2}$ Division of Brain Sciences, Department of Physiology, Faculty of Medicine, Kurume University School of \\ Medicine, Kurume, Japan \\ Email: nojiri@kumamoto-hsu.ac.jp
}

Received 30 September 2014; revised 17 November 2014; accepted 2 December 2014

Copyright (C) 2014 by authors and Scientific Research Publishing Inc.

This work is licensed under the Creative Commons Attribution International License (CC BY). http://creativecommons.org/licenses/by/4.0/

(c)

\begin{abstract}
In an attempt to study the relationship between sleep postures and sleep parameters assessed by actigraphy, we applied the newly developed Activity Monitoring and Evaluation System (A-MES) and actigraphy at the same time to younger and senior Japanese volunteer groups. It was found that sleep postures and diurnal activity determines, to some extent, sleep parameters including activity mean score (AMS), activity index (ACTX), waking episodes (WEP) and sleep fragmentation index (SFX). It was also found that sleep properties are different in younger and senior Japanese. For example, increase in the proportion of time in the supine position resulted in enhancement and deterioration of the sleep in the younger and senior groups, respectively. Furthermore, there were correlations between supine posture and AMS, ACTX, SFX, total minutes scored as awake (TMSA) and WEP obtained by actigraphy in the younger group, but only AMS and ACTX in senior group. In addition, we also assessed sleep parameters by use of questionnaires, and found that objective sleep quality was rather poor but subjective sleep quality was better in the senior group. In the younger group, in contrast, objective sleep quality was better but subjective sleep quality was poor. On the other hand, there was no correlation between sleep parameters assessed by actigraphy and self-report at all. The present study with A-MES and actigraphy provides the first evidence that sleep posture affects sleep quality and is a convenient, inexpensive and home-based method for studying sleep.
\end{abstract}

\section{Keywords}

Actigraphy, Activity Monitoring and Evaluation System (A-MES), Sleep Posture, Sleep Quality, Good Sleepers

How to cite this paper: Nojiri, A., Okumura, C. and Ito, Y. (2014) Sleep Posture Affects Sleep Parameters Differently in Young and Senior Japanese as Assessed by Actigraphy. Health, 6, 2934-2944. 


\section{Introduction}

Sleep evaluation in humans has been performed with polysomnography (PSG), a technique considered the "gold standard" for sleep studies. However, subjects have to stay in the sleep laboratory for at least four nights with many electrodes and sensors. As an alternative to PSG, the use of wrist actigraphy (activity-based monitoring), an instrument that measures wrist motor activity to provide an indirect assessment of sleep through the use of algorithm, has become an essential tool in sleep research and sleep medicine [1] [2]. However, it is not feasible to assess sleep postures per se by use of actigraphy, and till now little study has been carried out on the relation between sleep postures and sleep parameters assessed by actigraphy.

It was first noticed, from the analysis of the sleep characteristics and sleep positions of good and poor sleepers [3] [4], assessed with a Super 8 Camera taking one frame every 8 seconds providing 3600 frames over 8 hours, that sleep positions constitute an important sleep parameter that may be related to the sleep quality.

In a previous study, by use of a newly developed Activity Monitoring and Evaluation System (hereafter referred as A-MES) [5] [6], we observed postures, posture shifts, duration of postural immobility and frequency of body movements during the time in bed of four Japanese age groups [7]. The reliability made by A-MES on postures and movements was examined by the comparison with those obtained by video-recording the behavior of subjects, and the results obtained with A-MES yielded good agreement with those obtained by analysis of videotape recordings [8]. The subjects slept two or three consecutive nights at home wearing night clothes with two A-MES attached to trunk and left thigh. This method allows natural and undisturbed sleep in the subject's own bed and gives reliable data through one to two nights recording. We found that there is significant ontogenetic decrease in supine position, and corresponding increase in sleep posture lying on the right, although there was no difference in the number of posture shifts or the duration and frequency of the postural immobility across the four age groups. A-MES provides a convenient, inexpensive and home-based method for studying putative sleep movement. However, a limitation of this method was that it was not possible to distinguish awake and sleep states [7]. Therefore in the present study, we applied actigraphy and A-MES at the same time to younger and senior Japanese subjects in an attempt to study the relationship between sleep postures and sleep parameters. In addition, to assess the subjective sleep quality, we used Pittsburg Sleep Quality index [9] and Oguri-Shirakawa-Azumi Sleep Inventory MA version [10] (hereafter referred as PSQI and OSA-SI-MA, respectively).

Thus, the aim of the present study was threefold. Firstly we performed a comparative study on sleep postures and activities during 24 hours in younger and senior Japanese groups by use of A-MES and actigraphy at the same time. Secondly, we studied the relationship between sleep posture and diurnal activities with the sleep parameters obtained by actigraphy. Finally, we studied the relationship, if any, between subjective and objective sleep quality.

\section{Methods}

\subsection{Subjects}

16 subjects (eight female and eight male) in each of the following age groups were studied: the younger group ranged between 21 - 23 years old $(\mathrm{m}=21.8 \pm 0.8(\mathrm{SD})$ ), and the senior group ranged between 66 - 84 years old $(\mathrm{m}=74.3 \pm 5.8(\mathrm{SD}))$. Table 1 shows socio-demographic characteristics of the subjects. The subjects were recruited by advertisements made by the university.

The inclusion criteria were: healthy, no physical handicap and no sleep disorder. The subjects were interviewed by A.N. and C.O. to confirm that they were normal and free from sleep and medical disorders. All subjects were fully aware of the present study on sleep and were actively willing to participate in the study. Senior or younger subjects slept for 5 to 6 or 2 to 3 consecutive nights respectively at home, and records of 2 consecutive nights for each subject were analyzed according to the previous studies [7].

It is known that sleeping postures vary among Japanese subjects but each subject has similar sleeping postures and position shifts at similar frequencies [11] [12]. Therefore, two similar records, taken when the subject felt they had slept well, were used for analysis.

The study was approved by the ethics committee of Kumamoto Health Science University on clinical experimentation.

\subsection{Recording of Activity and Positions during $24 \mathrm{~h}$ by A-MES}

Activity Monitoring and Evaluation System (A-MES) [5] [6], a commercially available product (Solid Brains, 
Table 1. Socio-demographic characteristics of the subjects.

\begin{tabular}{ccc}
\hline \multirow{2}{*}{ Characteristics } & \multicolumn{2}{c}{ Number of subjects } \\
\cline { 2 - 3 } Age (years) & Younger group & Senior group \\
$21-23$ & 16 & 16 \\
$66-84$ & & \\
Gender & 8 & 8 \\
Male & 8 & 8 \\
Female & & 16 \\
Marital status & & \\
Married & 16 & \\
Never married & & \\
Employment & 14 & 2 \\
Student & 2 & 10 \\
Fulltime & & \\
Part-time & & \\
Retired & & \\
Ethnicity & 16 & \\
Japanese & &
\end{tabular}

2081-10 Tabaru, Mashiki-machi Kamimasikigun Kumamoto 861-2202, Japan), was used in the present study. A-MES consists of three-dimensional acceleration sensors $(27.2(\mathrm{D}) \times 27.2(\mathrm{~W}) \times 19.8(\mathrm{H}) \mathrm{mm})$ and a data logger. DC and AC components of signals of the three-dimensional acceleration sensors (sampling rate 5/second) detect the posture and movements of the subject. By use of A-MES attached to the trunk and left thigh of the subjects, it is possible to judge the following postures lying (supine, on the right or left side or prone), sitting, standing-up, walking and driving a wheelchair by the combination of the signals from trunk and left thigh [7].

The size and weight (28 g) are compact enough to attach to the subject's trunk or thighs. In the present study, however, we inserted A-MES into two pockets attached to the breast and left thigh of underwear (M, L and LL sizes) to monitor the postures and posture shifts during $24 \mathrm{~h}$. The records were taken on two or three consecutive days. The subjects were asked to bring back the underwear and A-MES for the analysis of the data.

\subsection{Actigraphy}

The subjects were also asked to wear the actigraphy on the non-dominant wrist. The actigraphy used was the Mini Motion logger Actigraph-Basic32C (Ambulatory Monitoring Inc., Ardsley, USA). Data were collected on the "zero crossing mode", in 1-min periods, and were scored on Action W-2 version 2.6.9905 software (Ambulatory Monitoring Inc., Ardsley NY). The algorithm is the most commonly reported analysis, and the following sleep parameters were analyzed: TMSA, total sleep time (TST), wake after sleep onset (WASO), sleep efficiency (SE), sleep onset latency (SOL), AMS, ACTX, SFX, wake episode (WEP) and long wake episodes (LWEP). Computerized analyses were performed according to the algorithms proposed by Cole et al. and Sadeh et al. for the estimation of actigraphic sleep parameters [13] [14]. The algorithm developed by Cole et al. yielded an 88\% agreement rate with PSG [13]. There have been numerous studies showing good concordance for sleep/wake estimation between actigraphy and PSG [15]-[22]. In normal sleepers, agreement coefficients between PSG and actigraphy of 0.90 and above have been reported [14].

\subsection{Questionnaire}

At the end of the study, we asked the subjects to assess OSA-SI-MA [10] which is a psychological measure to evaluate the sleep inspection at the time of rising. The inventory consists of sixteen items (choice of one out of four alternatives for each item) into five factors: 1) sleepiness on rising, 2) initiation and maintenance of sleep, 3) dreaming frequency, 4) refreshed feeling, 5) sleep length. The mean score is taken as 50 points for each factor, 
and the larger score indicates better sleep quality. We also used PSQI [9], a self-rated questionnaire which assesses sleep quality and disturbances over a 1-month period. Nineteen individual items provide seven "component” scores: subjective sleep quality, sleep latency, sleep duration, habitual sleep efficiency, sleep disturbance, use of sleeping medication and daytime dysfunction. The sum of scores for these seven components yields one global score, and the smaller the score the better the sleep quality. Scores more than six indicate sleep disorder of the subjects.

\subsection{Data Analysis}

We used Mann-Whitney's U test to analyze the proportion of time for each posture (lying, sitting, standing and walking) during 24 hours, sleep postures (supine, lying on right or left side, or prone) and sleep parameters (AMS, TMSW, SE, SOL, ACTX, WASO and LWASO) in the two age groups. For analysis of correlation between sleep posture or diurnal activity and various parameters in the two age groups, Spearman's correlation coefficient rank test was used. The difference in the ratio of sleep postures to the total time in bed was examined by use of the Steel-Dwass test. Values of $p<0.05$ were considered significant.

\section{Results}

\subsection{Postures in 24 Hours}

Postures in 24 hours were assessed by A-MES in the younger and senior groups. Figure 1 shows that lying and sitting were the main postures in both groups during 24 hours, and that standing and walking were more dominant in the senior than the younger group, whereas sitting was significantly longer in the younger group.

\subsection{Postures and Posture Shifts in Sleep}

We applied A-MES and actigraphy at the same time, to enable us to study the postures in sleep (Figure 2(a)). In general, four different postures, namely supine, lying on the right or left side, and prone, were observed in the two groups. The main postures in sleep were, in order, supine $>$ lying on the right or on the left $>$ prone in both groups, and the proportion of time in supine and prone postures were greater in the younger than the senior group. However, the proportion of time lying on the right was significantly larger in the senior than the younger group. In addition, the number of posture shifts was significantly greater in the younger group (Figure 2(b)).

\subsection{Objective Sleep Parameters}

In an attempt to assess the objective sleep quality, we used various parameters obtained by actigraphy.

Table 2 shows TMSA, TST, WASO, SE, SOL, AMS, ACTX, SFX, WEP and LWEP observed in the younger and senior groups. The scores for TMSA, AMS, ACTX and LWASO were larger in the senior than the younger group. On the contrary, scores for SE were higher in the younger than the senior group. These observations indicate that the objective sleep parameters in the younger were better than in the senior group.

\subsection{Relationship between Sleep Postures and Sleep Parameters}

In an attempt to study the relationship between the sleep postures and sleep parameters in the two groups, firstly we observed the relationship between supine posture and sleep parameters obtained by actigraphy. Interestingly, there was a positive correlation between supine postures and AMS or ACTX in the senior group and a negative correlation in the younger group. Increases in the proportion of time in the supine posture resulted in decrease and increase in AMS or ACTX in the senior and younger groups respectively (Figures 3(a)-(d)). Furthermore in the younger group, there were negative correlations between the proportion of time in the supine posture and SFX, WEP and TMSA (Figure 4), thereby indicating that the increases in the proportion of time in the supine posture resulted in enhancement of the sleep in the younger group.

\subsection{Posture Shifts and Sleep Parameters}

As shown in Table 3, there were also positive correlations between number of posture shifts and ACTX or WEP in younger, and TMSA, WASO, SE, ACTX, SFX or WEP in senior groups, respectively. 


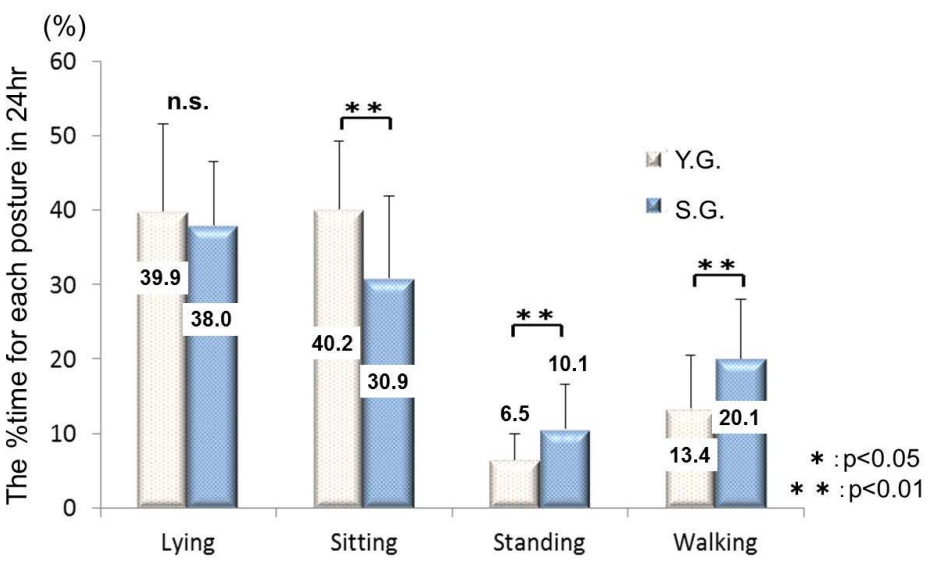

Figure 1. Postures (lying, sitting, standing and walking) during 24 hours assessed by A-MES in the younger and senior groups. S.G.: senior group, Y.G.: younger group.
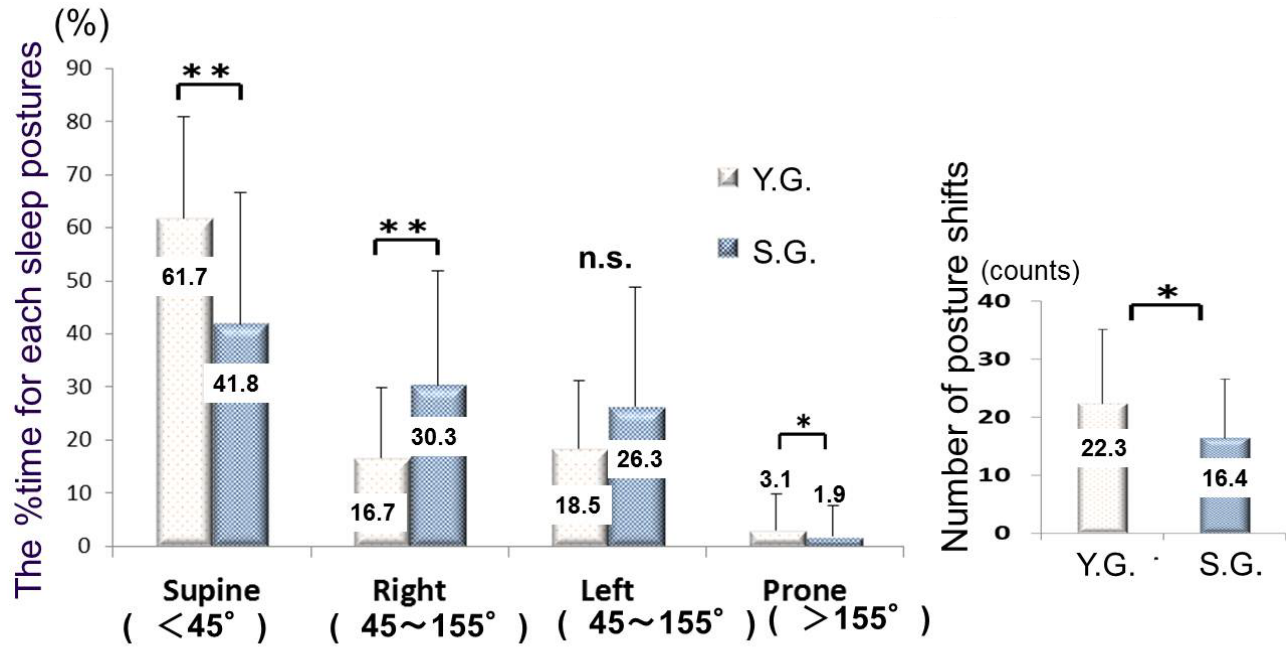

(a)

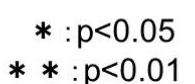

(b)

Figure 2. Postures (supine, lying on the right or left, and prone) in sleep assessed by actigraphy and A-MES at the same time, and posture shifts during sleep at night in younger and senior groups. S.G.: senior group, Y.G.: younger group.

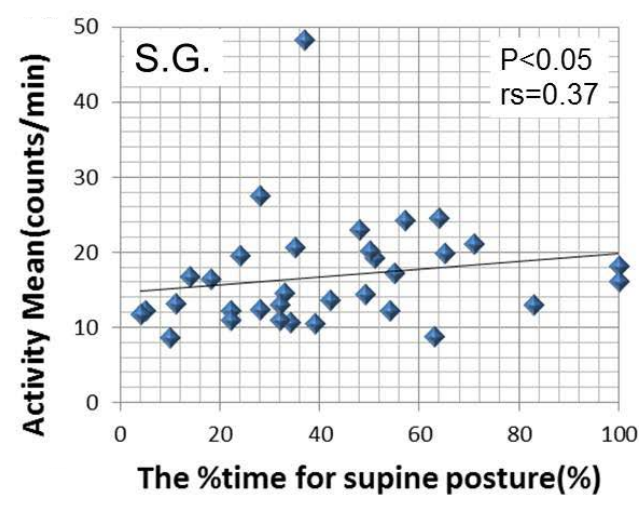

(a)

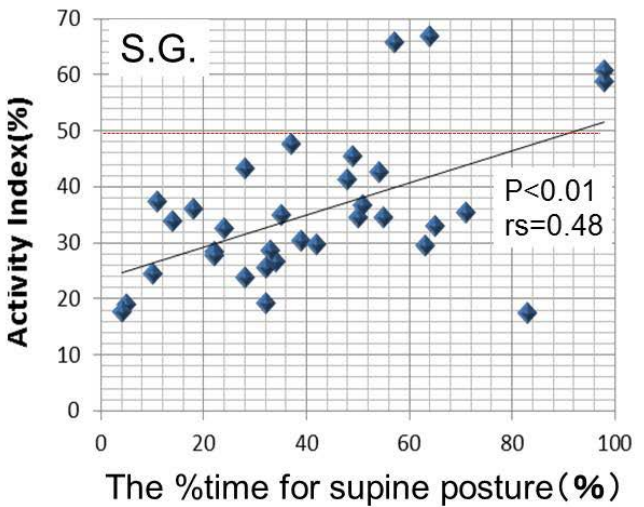

(b) 


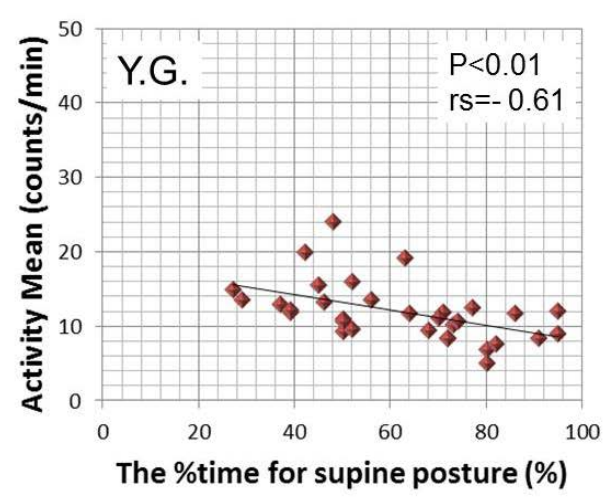

(c)

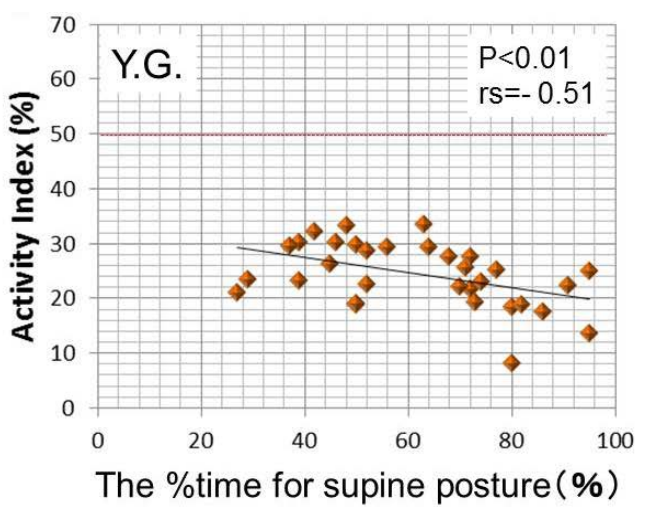

(d)

Figure 3. Relationships between the proportion of time in the supine posture in sleep and activity mean score (AMS) ((a) \& (c)) or activity index (ACTX) ((b) \& (d)). (a) \& (b), (c) \& (d): there were positive and negative correlations in senior and younger groups, respectively. S.G.: senior group, Y.G.: younger group.

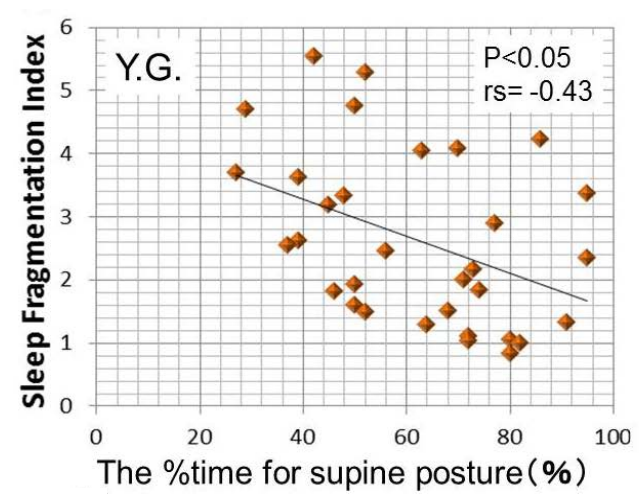

(a)

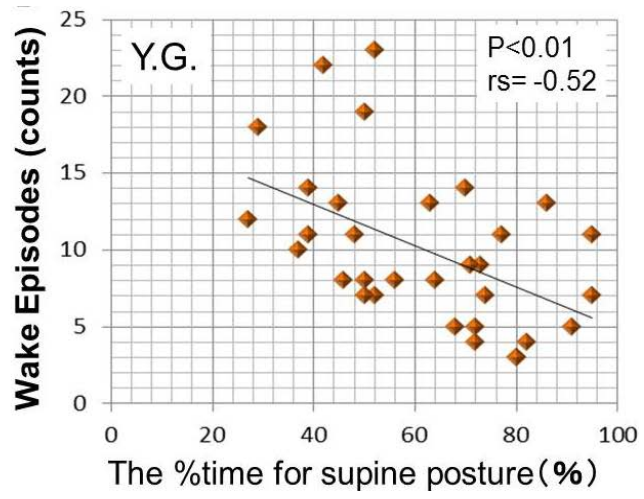

(b)

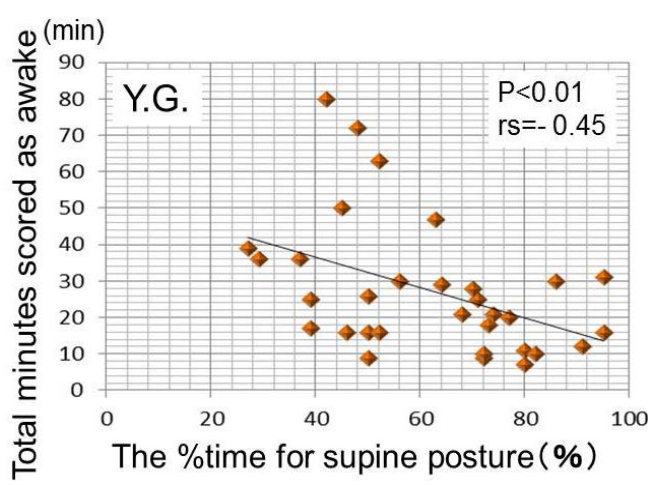

(c)

Figure 4. Relationships between the proportion of time in supine posture in sleep with (a): sleep fragmentation index (SFI) and (b): wake episode (WEP) and (c): total minutes scored as awake (TMSA) in the younger group. There were negative correlations for the three relationships. The coefficient values were $-0.43,-0.52$ and -0.45 , respectively. Y.G.: younger group.

\subsection{Diurnal Activity and Sleep Parameters}

We found negative correlations between the AMS or proportion of time walking during the day and ACTX in the senior group (Figure 5), indicating that daytime activities enhanced the sleep in the senior group. However, there was no correlation between diurnal activities and sleep parameters in the younger group. 
Table 2. Objective sleep parameters (total minutes scored as awake (TMSA), total sleep time (TST), wake after sleep onset (WASO), sleep efficiency (SE), sleep onset latency (SOL), activity mean score (AMS), activity index (ACTX), sleep fragmentation index (SFX), wake episode (WEP) and long wake episodes (LWEP)) assessed by actigraphy in younger and senior groups.

\begin{tabular}{|c|c|c|c|}
\hline & Younger group & Senior group & \\
\hline Total minutes scored as awake (TMSA) (min) & $27.4 \pm 18.2$ & $41.8 \pm 28.8$ & ${ }^{* *}$ \\
\hline Total sleep time (TST) (min) & $388.7 \pm 69.0$ & $375.0 \pm 61.3$ & n.s. \\
\hline Wake after sleep on set (WASO) (min) & $20.1 \pm 17.1$ & $32.0 \pm 26.1$ & $* *$ \\
\hline Sleep efficiency (SE) (\%) & $95.1 \pm 3.9$ & $92.3 \pm 5.3$ & $* *$ \\
\hline Sleep on set latency (SOL) (min) & $6.3 \pm 4.8$ & $7.5 \pm 10.3$ & n.s. \\
\hline Activity mean score (AMS) (counts/min) & $12.0 \pm 3.9$ & $16.8 \pm 7.4$ & ** \\
\hline Activity index (ACTX) (\%) & $24.4 \pm 5.8$ & $35.4 \pm 12.9$ & ** \\
\hline Sleep fragmentation Index (SFX) & $2.6 \pm 1.4$ & $2.9 \pm 1.7$ & n.s. \\
\hline Wake episodes (WEP) (counts) & $10.1 \pm 5.2$ & $10.2 \pm 4.4$ & n.s. \\
\hline Long wake episodes (LWEP) (counts) & $1.9 \pm 1.4$ & $2.6 \pm 1.3$ & $*$ \\
\hline
\end{tabular}

${ }^{*}: \mathrm{p}<0.05 ;{ }^{* *}: \mathrm{p}<0.01$.

Table 3. Relationships between the number of posture shifts in sleep and sleep parameters assessed by actigraphy in the younger and senior groups.

\begin{tabular}{ccc}
\hline Objective sleep parameters & \multicolumn{2}{c}{ Number of posture shifts } \\
\cline { 2 - 3 } Total minutes scored as awake (TMSA) & Younger group & Senior group \\
Wake after sleep on set (WASO) & n.s. & $0.36^{*}$ \\
Sleep efficiency (SE) & n.s. & $-0.48^{*}$ \\
Sleep on set latency (SOL) & n.s. & n.s. \\
Activity index (ACTX) & n.s. & $0.37^{*}$ \\
Sleep fragmentation Index (SFX) & $0.48^{* *}$ & $0.38^{*}$ \\
Wake episodes (WEP) & n.s. & $0.44^{*}$ \\
Long wake episodes (LWEP) & $0.38^{* *}$ & n.s. \\
\hline
\end{tabular}

${ }^{*}: \mathrm{p}<0.05 ;{ }^{* *}: \mathrm{p}<0.01$.

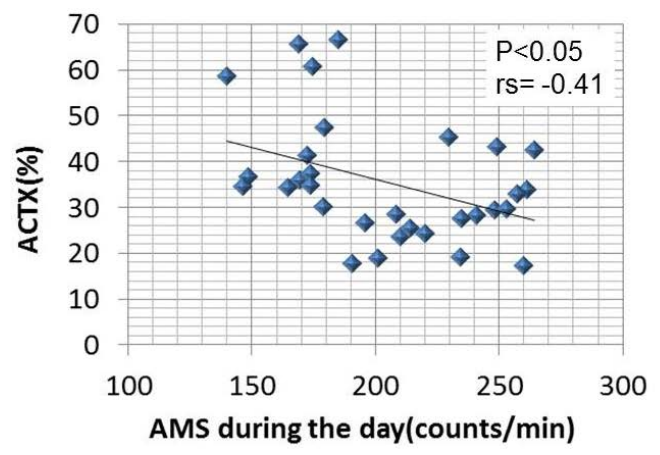

(a)

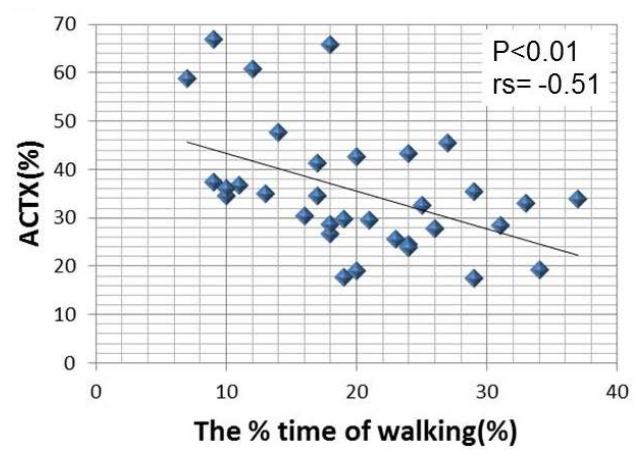

(b)

Figure 5. Relationships between activity mean score (AMS) (a) or the proportion of time for walking (b) with activity index (ACTX) in the senior group. All had a negative correlation. 


\subsection{Subjective Sleep Quality}

The subjective sleep quality was assessed by use of PSQI and OSA-SI-MA methods. As shown in Figure 6, the PSQI score was slightly larger in the younger than the senior group. However all OSA scores were larger in the senior than the younger group, thereby indicating that subjective sleep quality is higher in the senior than the younger group.

\subsection{Relationship between Objective and Subjective Sleep Quality}

In an attempt to study the correlation, if any, between objective and subjective sleep quality, we plotted sleep parameters (TMSA, WASO, SE, SOL, ACTX, SFX, WEP and LWEP) obtained by actigraphy against PSQI and OSA-SI-MA scores. However, there were no correlations between those parameters in either the younger or the senior groups (data not shown).

\section{Discussion}

The present results could be summarized as follows; 1 ) the main postures observed by A-MES in 24 hrs were lying or sitting in both the senior and younger groups, 2) the proportion of time in the sleep postures were in the order supine $>$ lying on the right, or left side > prone both groups, 3) there were correlations to some extent between sleep posture and sleep parameters obtained by actigraphy including AMS, ACTX, SFX and WEP, 4) there were correlations between sleep parameters and posture shifts in both younger and senior groups, and diurnal activity in the senior group, 5) objective sleep quality was better in the younger than the senior group, although subjective sleep quality was better in the senior group, 6) there was no correlation between sleep parameters assessed by actigraphy and self-report questionnaires.

In the previous study [7] we reported postures in bed by use of A-MES and found that there is an ontogenetic decrease in supine position. The present result confirmed this observation, and as shown in Figures 3-5 there were positive and negative correlations between sleep parameters assessed by actigraphy with postures, number of posture shifts and diurnal activity assessed by A-MES. Therefore, it is reasonable to assume that postures in bed during the night observed by A-MES represent sleep postures, suggesting that the recordings by two A-MES attached to the trunk and left thigh could fulfill the minimum requirement to study sleep postures.

In the present study we found different sleep properties in younger and senior Japanese, and the differences are as followings; 1) there were correlations between the proportion of time in the supine posture in sleep and AMS, ACTX, SFX, TMSW and WEP obtained by actigraphy in the younger group, but only AMS and ACTX in senior group, 2) increase in the proportion of time in the supine posture enhanced the sleep parameters including AMS, ACTX, SFX and WASO in the younger group, but reduced these parameters in the senior group, 3) increase in the number of the posture shifts during sleep decreased the sleep parameters such as SFX and WASO in the senior group, but showed almost no effects in the younger group even though the number of position shifts were larger than in the senior group, 4) objective sleep quality was rather poor but subjective sleep

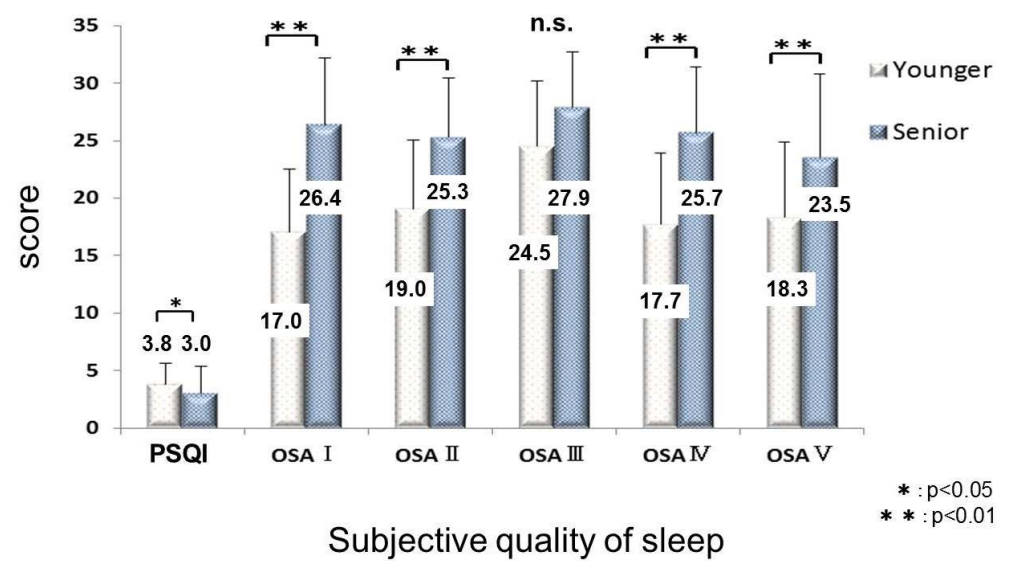

Figure 6. Subjective sleep quality assessed by PSQI or OSA-SI in younger and senior groups. 
quality was better in the senior group; in contrast, in the younger group objective sleep quality was better but subjective sleep quality was poor. The exact mechanisms involved in these different results are yet to be clarified. However, the present results indicate for the first time that sleep posture affects the sleep parameters obtained by actigraphy in younger and senior Japanese, and that sleep parameters might be one of the targets for aging.

We also found that there was no correlation between sleep parameters assessed by actigraphy and self-report questionnaires. However, self-report remains the most practical and cost-effective method in epidemiologic sleep studies attempting to collect information on large population-based samples, and it was found that a 3-item sleep questionnaire of the type typically used in epidemiologic studies show poor agreement with actigraphically recorded sleep habits [23]. Similarly, the skin condition of children with atopic dermatitis before and after treatment with pimecrolimus, an immunomodulating agent, was related to sleep parameters obtained by actigraphy, but subject perception of itching was not correlated with any sleep parameters [24].

On the other hand, there are reports which indicate correlations to some extent between subjective and objective sleep indices. Firstly, it was proposed that the probabilistic sleep mode (PSM) obtained by processing 3-second EEGs represents the sleep profile (not only the sleep stage but also sleep quality) and is correlated with psychometric tests [25]. Significant correlations between sleep architecture and dynamic variables of sleep quality have also been reported [26]. Secondly, the relationship between PSG and subjective sleep quality has been directly investigated [27] and it was found that the appearance duration/ratio of sleep stage II/III/IV shows a significant correlation with each OSA-SI-MA factor score. Thus, the authors claimed that they could estimate the OSA-SI-MA factor from hypnogram indices with maximum accuracy of $71 \%$ by use of artificial neural network.

Recently, the feasibility and acceptability of actigraphy to monitor sleep quality and quantity was examined in healthy self-rated good sleeping adults at home-based settings. Actigraphy data were used to prepare an individual sleep report and participants were also asked to complete a Self-report Health Related Quality of Life (HRQL) using the WHO five Well Being Index (WBI) questionnaire. Although all participants in the study were self-rated healthy and "good sleepers", there were cases where low sleep quality were noticed. Thus, it seems that actigraphy can be easily utilized to monitor sleep-wake patterns at home. Furthermore, a discrepancy between self-reported and actigraphic sleep quality was also reported [28].

These various results and the present observations, taken together, indicate that further studies are warranted to understand the relationship between objective and subjective sleep obtained by the various methods. Furthermore, it seems essential to examine the consistency of the results obtained by multiple measures of sleep parameters.

Till now, surprisingly little study has been carried out on the relationship between sleep posture and sleep quality, and the present observations indicate that sleep postures constitute an important sleep variable which is related to sleep quality. The present study with A-MES and actigraphy provides a convenient, inexpensive and home-based method for studying sleep.

\section{Conclusion}

By application of newly developed A-MES and actigraphy to younger and senior Japanese volunteer groups at the same time, we obtained following conclusions. Firstly, sleep postures and diurnal activity determine, to some extent, sleep parameters including AMS, ACTX, WEP and SFX assessed by actigraphy, and this is the first evidence that sleep posture affects sleep quality. Secondly, sleep properties are different in younger and senior Japanese, including the relationship between the proportion of time in the supine position and sleep quality, or AMS, ACTX, SFX, and TMSA. Thirdly, we also assessed sleep parameters by use of questionnaires; however there was no correlation between sleep parameters assessed by actigraphy and self-report at all. Finally, present study with A-MES and actigraphy provides a convenient, inexpensive and home-based method for studying sleep.

\section{Acknowledgements}

We thank Dr. Kate E. Creed for critical reading of the manuscript and pertinent comments. This work was supported by Grant-in-Aid from Kumamoto Health Science University for AN \& YI. 


\section{References}

[1] Ancoli-Israel, S., Cole, R., Alessi, C., Chambers, M., Moorcroft, W. and Pollak, C.P. (2003) The Role of Actigraphy in the Study of Sleep and Circadian Rhythms. Sleep, 26, 342-392.

[2] Mogenthaler, T., Alessi, C., Friedman, L., et al. (2007) Practice Parameters for the Use of Actigraphy in the Assessment of Sleep and Sleep Disorder: An Update for 2007. Sleep, 30, 519-529.

[3] De Koninck, J.M., Gagnon, P. and Lallier, S. (1983) Sleep Positions in the Young Adult and Their Relationship with the Subjective Quality of Sleep. Sleep, 6, 52-59.

[4] De Koninck, J.M., Lorrain, D. and Gagnon, P. (1992) Sleep Positions and Position Shifts in Five Age Groups: An Ontogenic Picture. Sleep, 15, 143-149.

[5] Sakata, S., Nagata, M. and Nojiri, S. (2002) Approach to the Measurement of ADL (Activities of Daily Living). DenO-Ken Technical Report, 12, 19-25.

[6] Sakata, S., Nojiri, S. and Nagata, M. (2004) The Improvement on the A-MES (Activity Monitoring and Evaluation System) for Commercialization. Den-O-Ken Technical Report, 14, 10-14.

[7] Nojiri, A., Kimura, I., Okumura, C., Akaike, A. and Ito, Y. (2012) Postures and Position Shifts in Bed Assessed with Three-Dimensional Acceleration Sensors Worn by Four Japanese Age Groups. Polish Journal of Rehabilitation Research, 2, 40-48.

[8] Kitamura, N., Sato, T., Kawagoshi, A., et al. (2010) Evaluation of Physical Activity in the Daily Life of Healthy Subjects with Special Reference to the Reliability and Validity of IPAQ as Evaluated by a Triaxial Accelerometer. Rigakuryoho Kagaku, 25, 767-771. http://dx.doi.org/10.1589/rika.25.767

[9] Buysse, D.J., Reynolds 3rd, C.F., Monk, T.H., Berman, S.R. and Kupfer, D.J. (1989) The Pittsburgh Sleep Quality Index: A New Instrument for Psychiatric Practice and Research. Psychiatry Research, 28, 193-213. http://dx.doi.org/10.1016/0165-1781(89)90047-4

[10] Yamamoto, Y., Tanaka, H., Takase, M., Yamasaki, K., Azumi, K. and Shirakawa, S. (1999) Standardization of Reviced Version of OSA Sleep Inventory for Middle-Aged and Aged. Brain Science and Disorders, 10, 401-409.

[11] Kubota, T., Kunisawa, N., Murayama, N., et al. (2001) Characteristics of Sleeping Positions in Healthy Male Adults. Reports of the Institute of Biomaterials and Bioengineering, 35, 35-41.

[12] Kubota, T., Ohshima, N., Kunisawa, N., Murayama, R., Okano, S. and Mori-Okamoto, J. (2003) Characteristic Features of the Nocturnal Sleeping Posture of Healthy Men. Sleep and Biological Rhythms, 1, 183-185. http://dx.doi.org/10.1046/j.1446-9235.2003.00040.x

[13] Cole, R.J., Kripke, D.F., Gruen, W., Mullaney, D.J. and Gillin, J.C. (1992) Automatic Sleep/Wake Identification from Wrist Activity. Sleep, 15, 461-469.

[14] Sadeh, A., Sharkey, K.M. and Carskadon, M.A. (1994) Activity-Based Sleep-Wake Identification: An Empirical Test of Methodological Issues. Sleep, 17, 739-743.

[15] Blackwell, T., Redline, S., Ancoli-Israel, S., Schneider, J.L., Surovec, S., Johnson, N.L., et al. (2008) Comparison of Sleep Parameters from Actigraphy and Polysomnography in Older Women: The SOF Study. Sleep, 31, $283-291$.

[16] Blackwell, T., Ancoli-Israel, S., Redline, S. and Stone, K.L. (2011) Factors That May Influence the Classification of Sleep-Wake by Wrist Actigraphy: The MrOS Sleep Study. Journal of Clinical Sleep Medicine, 7, 357-366.

[17] De Souza, L., Benedio-Silva, A.A., Pires, M.L.N., Poyares, D., Tufik, S. and Calil, H.M. (2003) Further Validation of Actigraphy for Sleep Studies. Sleep, 26, 81-85.

[18] Hedner, J., Pillar, G., Pittman, S.D., Zou, D., Grote, L. and White, D.P. (2004) A Novel Adaptive Wrist Actigraphy Algorithm for Sleep-Wake Assessment in Sleep Apnea Patients. Sleep, 27, 1560-1566.

[19] Hyde, M., O’Driscoll, D.M., Binette, S., Galang, C., Tan, S.K., Verginis, N., et al. (2007) Validation of Actigraphy for Determining Sleep and Wake in Children with Sleep Disordered Breathing. Journal of Sleep Research, 16, 213-216. http://dx.doi.org/10.1111/j.1365-2869.2007.00588.x

[20] Johnson, N.L., Kirchner, H.L., Rosen, C.L., Storfer-Isser, A., Cartar, L.N., Ancoli-Israel, S., et al. (2007) Sleep Estimation Using Wrist Actigraphy in Adolescents with and without Sleep Disordered Breathing: A Comparison of Three Data Modes. Sleep, 30, 899-905.

[21] Jean-Louis, G., Kripke, D.F., Cole, R.J., Assmus, D.J. and Langer, R.D. (2001) Sleep Detection with an Accelerometer Actigraph: Comparison with Polysomnography. Physiology \& Behavior, 72, 21-28. http://dx.doi.org/10.1016/S0031-9384(00)00355-3

[22] Lichstein, K.L., Stone, K.C., Donaldson, J., Nau, S.D., Soeffing, J.P., Murray, D., et al. (2006) Actigraphy Validation with Insomnia. Sleep, 29, 232-239.

[23] Girschik, J., Fritschi, L., Heyworth, J. and Waters, F. (2012) Validation of Self-Reported Sleep against Actigraphy. 
Journal of Epidemiology, 22, 462-468. http://dx.doi.org/10.2188/jea.JE20120012

[24] Leo, H.L., Bender, B.L., Leung, S.B., Tran, Z.V. and Leung, D.Y.M. (2004) Effect of Pimecrolimus Cream 1\% on Skin Condition and Sleep Disturbance in Children with Atopic Dermatitis. Journal of Allergy and Clinical Immunology, 114, 691-693. http://dx.doi.org/10.1016/j.jaci.2004.05.037

[25] Lewandowski, A., Rosipal, R. and Dorffner, G. (2012) Extracting More Information from EEG Recordings for a Better Description of Sleep. Computer Methods and Programs in Biomedicine, 108, 961-972. http://dx.doi.org/10.1016/j.cmpb.2012.05.009

[26] Rosipal, R., Lewandowski, A. and Dorffner, G. (2013) In Search of Objective Components for Sleep Quality Indexing in Normal Sleep. Biological Psychology, 93, 210-220. http://dx.doi.org/10.1016/j.biopsycho.2013.05.014

[27] Igasaki, T., Taniyama, Y., Matsuda, Y. and Murayama, N. (2013) Can “Quality of Sleep” Be Evaluated from Hypnogram?-Estimation of Factor Score of Oguri-Shirakawa-Azumi Sleep Inventory by Artificial Neural Network. Proceedings of the Information Systems International Conference (ISICO), Bali, 2-4 December 2013, 373-378.

[28] Noor, Z.M., Smith, A.J., Smith, S.S. and Nissen, L.M. (2013) Feasibility and Acceptability of Wrist Actigraph in Assessing Sleep Quality and Sleep Quantity: A Home-Based Pilot Study in Healthy Volunteers. Health, 5, 63-72. http://dx.doi.org/10.4236/health.2013.58A2010 
Scientific Research Publishing (SCIRP) is one of the largest Open Access journal publishers. It is currently publishing more than 200 open access, online, peer-reviewed journals covering a wide range of academic disciplines. SCIRP serves the worldwide academic communities and contributes to the progress and application of science with its publication.

Other selected journals from SCIRP are listed as below. Submit your manuscript to us via either submit@scirp.org or Online Submission Portal.
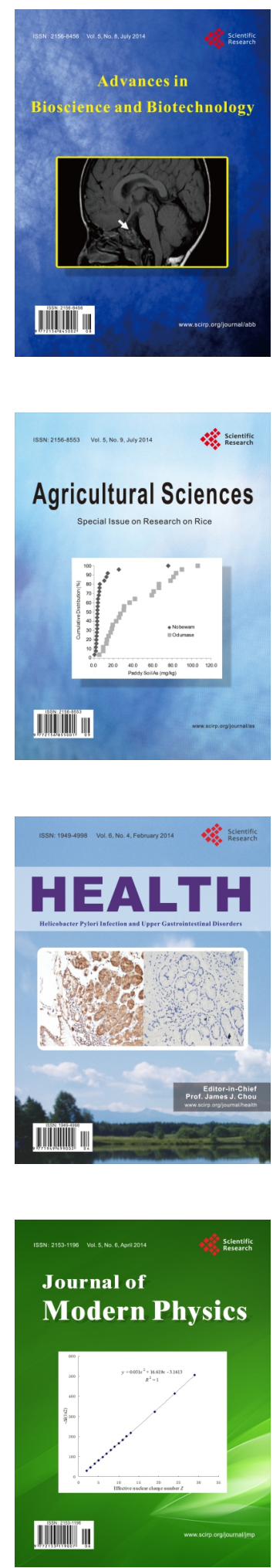
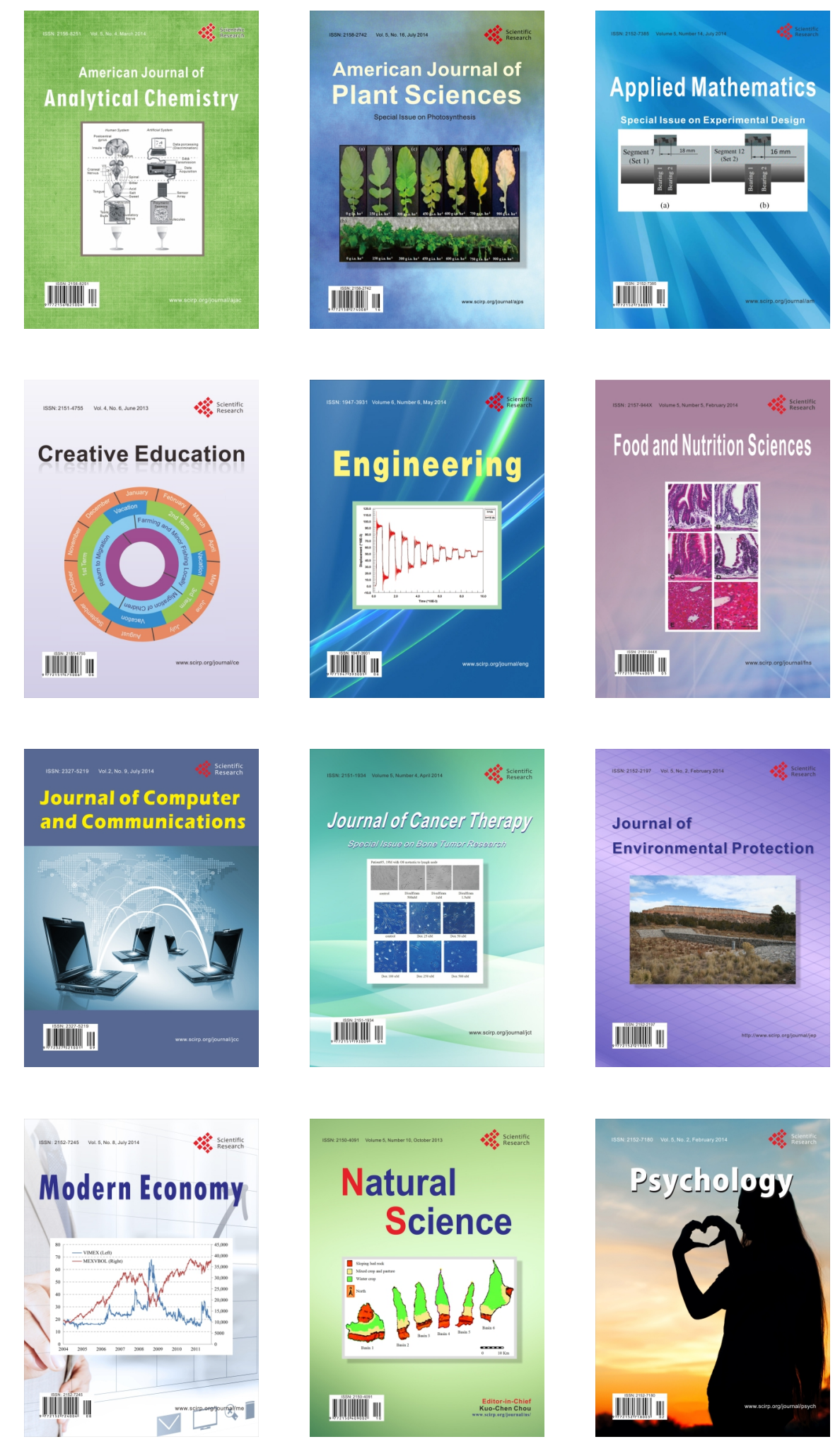